\section{\#124 Fluorose e Cárie Dentária na Ilha de São Miguel}

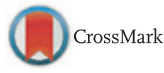

Rita Teixeira, Sónia Mendes*

FMDUL

Objetivos: A finalidade deste trabalho é contribuir para o estudo das doenças orais e dos comportamentos com estas relacionadas, nos jovens de 12 anos da Ilha de São Miguel. Os seus objetivos específicos são: estudar comportamentos de saúde oral e a prevalência de cárie e fluorose dentária, bem como os seus principais determinantes. Materiais e métodos: Foi utilizada informação previamente recolhida no III Estudo Nacional de Prevalência das Doenças Orais, realizado em 2012. Este estudo incluiu 145 indivíduos de 12 anos da Ilha de São Miguel. A recolha de dados incluiu um questionário e uma observação intraoral. Foram utilizados os critérios do International Caries Detection and Assessment System, e o índice de Dean. Foi realizada a análise descritiva e utilizados os testes de Qui-quadrado, Teste Exato de Fisher, Mann-Whitney e Kruskal-Wallis (nível de significância de 0,05). Resultados: A maioria dos participantes escovava os dentes duas ou mais vezes por dia $(72,4 \%)$ com dentífrico fluoretado $(44,1 \%)$, não usava fio dentário $(81,9 \%)$ e já tinha visitado um médico dentista (75,4\%). A prevalência de cárie foi $29,6 \%$ e o valor médio do CPOD foi 0,67. A prevalência de fluorose dentária foi de $24,1 \%$ e o valor médio do Índice de Dean foi 0,44. A cárie dentária revelou-se associada à visita ao médico dentista e a uma pior perceção do estado de saúde oral $(p<0,05)$. A fluorose dentária demonstrou-se associada ao nível de instrução da mãe $(p<0,05)$. Conclusões: A população demonstrou, de um modo geral, bons comportamentos de saúde oral. No que se refere à prevalência e gravidade de cárie dentária, os resultados revelaram, na ilha de São Miguel, um CPOD e índice médio de Dean baixos. Verificou-se uma associação positiva entre a cárie e a visita ao dentista e uma relação inversa com a perceção do estado de saúde oral. As crianças cujas mães tinham um nível de instrução mais alto apresentaram mais fluorose dentária. http://doi.org/10.24873/j.rpemd.2019.12.586

\#125 Qualidade de Vida relacionada com a Saúde Oral numa população portuguesa de 18 anos

Joana Ferreira da Costa, Sónia Mendes, Mário Bernardo*

Faculdade de Medicina Dentária da Universidade de Lisboa

Objetivos: Os objetivos deste estudo foram: a) relacionar a qualidade de vida relacionada com a saúde oral (QdVRSO) com o estado de saúde oral e comportamentos relacionados com a saúde oral; b) relacionar a autoperceção do estado de saúde oral com o estado de saúde oral e comportamentos relacionados com a saúde oral. Materiais e métodos: Foi utilizada informação previamente recolhida do III Estudo Nacional de Prevalência das Doenças Orais, realizado em 2013. A amostra foi constituída por 157 indivíduos com 18 anos de idade pertencentes à região de Lisboa e Vale do Tejo. A recolha dos dados foi realizada através de um questionário, que recolheu informações acerca da QdVRSO, utilizando 8 questões, retiradas do
OHIP-14 (Oral Health Impact Profile), e da autoperceção da saúde oral; e através de uma observação intraoral, que registou informações sobre o estado de saúde oral, nomeadamente presença de cárie dentária (critérios ICDAS II) e presença de hemorragia gengival (IPC modificado). Os comportamentos e outros aspetos relacionados com a saúde oral analisados foram o género, a área de residência, o nível de escolaridade do próprio, o nível de escolaridade da mãe, a escovagem bidiária, a visita a um profissional de saúde oral e os hábitos tabágicos. Foi realizada a análise descritiva dos dados e utilizados os testes de Mann-Whitney e Kruskal-Wallis $(\alpha=0,05)$. Resultados: A proporção de respostas 'Nunca' às 8 questões da QdVRSO, que correspondem a uma melhor qualidade de vida variaram entre $38,5 \%$ e $96,8 \%$. Quase metade $(47,2 \%)$ dos participantes referiram uma autoperceção da sua saúde oral "má" $(4,5 \%)$ ou "razoável” (42,7\%). A prevalência de cárie (C5-6POD10) foi de $54,8 \%$, e a prevalência de hemorragia gengival foi de $29,3 \%$. Conclusões: Verificou-se uma pior QdVRSO no sexo feminino, em indivíduos com presença de cárie, com um nível de escolaridade mais baixo, que não visitavam o profissional de saúde oral e com presença de hemorragia gengival. Os indivíduos que escovavam os dentes duas por dia, cujas mães tinham um nível de escolaridade superior, que nunca fumaram, sem presença de cárie e sem hemorragia gengival revelaram uma melhor autoperceção da sua saúde oral.

http://doi.org/10.24873/j.rpemd.2019.12.587

\#126 Caracterização de hábitos relacionados com a higiene oral numa população portuguesa

Ana Freitas Costa *, Inês Catarina A. Inocêncio, ML Pereira

Faculdade de Medicina Dentária da Universidade do Porto, Faculdade de Medicina Dentária da Universidade do Porto. EpiUnit.

Objetivos: O objetivo deste trabalho de investigação foi caracterizar os hábitos de higiene oral numa amostra de utentes na Faculdade de Medicina Dentária da Universidade do Porto. Materiais e métodos: A amostra deste estudo transversal era constituída por utentes da Faculdade em causa, sendo estes adultos e estavam presentes na sala de espera da Faculdade enquanto aguardavam a consulta. Como critério de exclusão consideramos pacientes em primeira consulta na Faculdade. A cada um foi feito um questionário em modo entrevista dividido em 2 partes. Na primeira efetuaram-se questões sociodemográficas (idade, sexo, entre outros). Na segunda recolheram-se dados relativos aos hábitos de higiene oral, uso de próteses/ aparelhos ortodônticos/ contenções e sua higienização e auto-perceção da saúde oral. A análise estatística foi feita com o IBM SPSS $25^{\circledR}$. As variáveis categóricas foram descritas através de frequências absolutas e relativas e as contínuas através da média e desvio padrão. Este estudo foi aprovado pela Comissão da Proteção de Dados Pessoais da Universidade do Porto, Comissão de Ética da Faculdade em causa e Comissão Científica do Mestrado Integrado de Medicina Dentária da mesma Faculdade. Para se caracterizar as atitudes de higiene oral adequada foi efetuado um score, atribuindo-se o valor 0 (não adequada) e 1 (adequada). Este último era atribuído a quem efetuava cor- 
retamente todos os passos da higiene oral (frequência, momento da escovagem, uso de auxiliares de higiene oral, escovagem da língua, uso de escova e dentífrico para a escovagem). Atribuíram-se os valores 0 (não adequado) e 1 (adequado) para cada passo e, depois, somaram-se estas variáveis. Resultados: Foram convidados a participar 190 pessoas voluntariamente, 16 pessoas recusaram e 174 aceitaram. A taxa de participação foi de $91,6 \%$. A maioria eram mulheres, eram casados e tinham mais do que 50 anos. 28\% frequentaram a Universidade e $39,7 \%$ eram reformados. A maioria visitava o médico dentista pelo menos uma vez por ano. A maioria escovava os dentes 2 ou mais vezes por dia. 46,8\% escovavam de manhã e à noite, 53,4\% escova a língua e 77\% usava auxiliares de higiene oral, sendo os mais usados o fio dentário e o colutório. Apenas 40,9\% apresentou uma higiene oral adequada. Conclusões: Estes dados poderão indicar a necessidade do aumento da literacia dos pacientes em saúde oral, através da promoção e educação para este tema e verificar se os pacientes entenderam as instruções fornecidas e reforçá-las em todas as consultas. http://doi.org/10.24873/j.rpemd.2019.12.588

\section{\#127 Hábitos relacionados com a saúde oral nos pacientes com tumores de cabeça e pescoço CrossMark}

Inês Catarina Alves Inocêncio*, Jorge Guimarães, André Soares, Ana Freitas Costa, Isabel Reis, ML Pereira

Faculdade de Medicina Dentária da Universidade do Porto, Instituto Português de Oncologia do Porto Francisco Gentil EPE, EpiUnit, Instituto de Saúde Pública da Universidade do Porto.

Objetivos: Avaliar os hábitos relacionados com a saúde oral em pacientes com patologia da cabeça e pescoço, submetidos a tratamentos de quimioterapia e/ou radioterapia e a importância que estes atribuem à saúde oral. Materiais e métodos: Neste estudo transversal, aplicou-se um questionário, por entrevista, a uma população adulta de 82 pacientes da Clínica de Cabeça e Pescoço do Instituto Português de Oncologia do Porto a realizar quimioterapia e/ou radioterapia. As questões incluíam o perfil sociodemográfico, estado de tratamento, hábitos de higiene oral, hábitos relacionados com a higiene oral, alterações na cavidade oral e autoperceção de saúde oral antes e depois do diagnóstico. Resultados: A média de idades dos participantes foi de 61,99 anos e a maioria era do sexo masculino. Relativamente aos hábitos de saúde oral, não se verificou um aumento significativo da frequência de escovagem. Quanto aos meios auxiliares de higiene oral, observou-se um aumento estatisticamente significativo da utilização de elixires e/ou colutórios. A escovagem das gengivas aumentou significativamente $(37,8 \%$ versus $48,8 \%$ ). Dos participantes, 59,8\% eram ex-fumadores, 15,9\% fumadores e 51,6\% deixou de fumar devido à doença. A maioria consumia álcool antes da doença, tendo 75,6\% dos doentes alterado esse consumo. Na autoperceção da higiene oral, observa-se um aumento estatisticamente significativo, na classificação como `Excelente/Boa“ após o diagnóstico. Xerostomia, disfagia e trismus foram as alterações mais sentidas. O oncologista é quem mais informa sobre saúde oral. Conclusões: O Médico Dentista deve educar para a saúde oral, enfatizando a impor- tância da manutenção da cavidade oral durante a doença, aconselhando estratégias que diminuam os efeitos adversos dos tratamentos e melhorem a qualidade de vida.

http://doi.org/10.24873/j.rpemd.2019.12.589

\#128 Erupção dentária e estatuto socioeconómico - importância nas estratégias preventivas

Santos MC*, Caldas IM, Pereira ML

FMDUP, CEF-UC INFACTS IUCS-Norte CESPU, EpiUnit-

-Instituto de Saúde Pública da Universidade do Porto

Objetivos: O objetivo deste estudo consistiu na caraterização do impacto clínico do estatuto socioeconómico na erupção dentária, numa perspetiva de adequação das estratégias preventivas da cárie dentária, tais como selantes de fissura e aplicações tópicas de flúor. Materiais e métodos: Classificou-se a erupção do segundo molar mandibular permanente, de acordo com o método de Olze et al., em 529 ortopantomografias selecionadas a partir de uma amostra previamente utilizada no estudo de Carneiro et al. A análise estatística foi realizada recorrendo ao software IBM Statistical Package for the Social Sciences $25^{\circledR}$. Efetuou-se a análise descritiva da distribuição do estadio de erupção de acordo com o estatuto socioeconómico, a idade e o sexo dos indivíduos. As idades, em cada estadio, foram comparadas de acordo com o grupo socioecónomico através do teste de Mann-Whitney U, utilizando o nível de significância 0,05. Resultados: Num total de 529 ortopantomografias de indivíduos com idades compreendidas entre os 3 e os 17 anos, 253 eram do sexo masculino e 276 do sexo feminino. Dos 529 indivíduos, $273(51,6 \%)$ pertenciam ao grupo socioeconomicamente desfavorecido e $256(48,4 \%)$ ao grupo socioeconomicamente favorecido. No sexo masculino não se verificaram diferenças estatisticamente significativas quando comparadas as idades por estadio, de acordo com o estatuto socioeconómico. O mesmo se verificou no sexo feminino, com exceção do estadio 1 ( $p=0,025)$. Conclusões: Os resultados apontam para a não existência de influência do estatuto socioeconómico no padrão temporal da erupção dentária do segundo molar mandibular permanente. Considerando o padrão de erupção observado, bem como as limitações deste estudo, poderá concluir-se que o momento da implementação de medidas preventivas da cárie dentária não será influenciado pelo estatuto socioeconómico. No entanto, estando descrito na literatura que a cárie dentária é mais prevalente associada a um estatuto socioeconómico mais baixo, a prevenção e promoção de saúde oral poderá ter de ser adequada ao estatuto socioeconómico do indivíduo. http://doi.org/10.24873/j.rpemd.2019.12.590

\#129 Avaliação do estado de saúde oral de atletas de alto rendimento - Atletismo

Andreia Crespo*, Sónia Mendes, Mário Filipe Bernardo

Faculdade de Medicina Dentária da Universidade de Lisboa

Objetivos: Os objetivos deste estudo foram: a) avaliar o estado de saúde oral de atletas de alto rendimento na modalida- 